\title{
Bad reporting does not mean bad methods for randomised trials: observational study of randomised controlled trials performed by the Radiation Therapy Oncology Group
}

Heloisa P Soares, Stephanie Daniels, Ambuj Kumar, Mike Clarke, Charles Scott, Suzanne Swann, Benjamin Djulbegovic

Department of
Interdisciplinary
Oncology, H Lee
Moffitt Cancer
Center and
Research Institute,
University of South
Florida, 12902
Magnolia Drive,
Tampa, FL 33612,
USA
Heloisa P Soares
research assistant
Ambuj Kumar
research associate
Benjamin
Djulbegovic
professor of oncology
and medicine
H Lee Moffitt
Cancer Center and
Research Institute
Stephanie Daniels
coordinator,Eastern
Cooperative Oncology
Group
UK Cochrane
Centre, Oxford
OX2 7LG
Mike Clarke
director
Statistical Unit,
Radiation Therapy
Oncology Group,
PA 19107, USA
Charles Scott
senior director
Suzanne Swann
senior biostatistician
Correspondence to:
B Djulbegovic
djulbebm@
moffitt.usf.edu

BMJ 2004;328:22-5

\begin{abstract}
Objective To determine whether poor reporting of methods in randomised controlled trials reflects on poor methods.

Design Observational study.

Setting Reports of randomised controlled trials conducted by the Radiation Therapy Oncology Group since its establishment in 1968.

Participants The Radiation Therapy Oncology Group.

Outcome measures Content of reports compared with the design features described in the protocols for all randomised controlled trials.

Results The methodological quality of 56 randomised controlled trials was better than reported. Adequate allocation concealment was achieved in all trials but reported in only $42 \%$ of papers. An intention to treat analysis was done in $83 \%$ of trials but reported in only $69 \%$ of papers. The sample size calculation was performed in $76 \%$ of the studies, but reported in only $16 \%$ of papers. End points were clearly defined and $\alpha$ and $\beta$ errors were prespecified in $76 \%$ and $74 \%$ of the trials, respectively, but only reported in $10 \%$ of the papers. The one exception was the description of drop outs, where the frequency of reporting was similar to that contained in the original statistical files of the Radiation Therapy Oncology Group.

Conclusions The reporting of methodological

aspects of randomised controlled trials does not necessarily reflect the conduct of the trial. Reviewing research protocols and contacting trialists for more information may improve quality assessment.
\end{abstract}

\section{Introduction}

Evaluation of the quality of published clinical research is central to informed decision making. Information on trial quality is particularly important during peer review or when results from individual studies are evaluated in systematic reviews or meta-analyses. ${ }^{12}$ The quality of research should always be considered when a report is used in decision making in health care. Poorly conducted and reported research seriously compromises the integrity of the research process, especially if biased results receive false credibility. ${ }^{3}$

Many efforts have been made to improve the quality of studies and their related publications. The best example was the publication of the Consolidated Standards of Reporting of Trials (CONSORT) statement to improve the quality of trial reports. ${ }^{3}$ Such efforts to improve the quality of clinical research, however, imply that if certain design or methodological features are not reported then they were not done. Ide- ally, assessment of the quality of clinical research should not only address reporting but also the original design and intended plan for its conduct and analysis as specified in the trial's research protocol. The importance of linking the final report of clinical trials with their original research protocols led some authors to argue that no randomised controlled trial should be conducted without publication of its research proto$\mathrm{col}^{4}{ }^{4}$ The reasons behind this are that critical comments may be encouraged leading to improvements in trial design, publication can be coupled with trial registration, the original protocol can be compared with what was subsequently done, and investigators can more easily appreciate what research is being conducted in their areas of interest. ${ }^{4}$ More importantly, publication of research protocols is one of the best ways to minimise bias by explicitly stating a priori hypotheses and methods without the prior knowledge of results. ${ }^{5}$ Many randomised controlled trials are preceded by the preparation of a written protocol, which describes the conduct of the trial more comprehensively than is possible in many journal articles, and making these protocols available would provide much useful additional information. We aimed to test the assumption that poor reporting reflects poor methods by comparing research protocols with the information published in the final reports of a set of randomised controlled trials.

\section{Methods}

We studied randomised controlled trials conducted by the Radiation Therapy Oncology Group. This is a national clinical cooperative group with a focus on the development of studies to improve survival and the quality of life of patients with cancer. It was established in 1968 and is publicly funded by the National Cancer Institute in the United States. The group consists of both clinical and laboratory investigators from over 260 institutions across the United States and Canada, and its membership includes nearly $90 \%$ of all comprehensive and clinical cancer centres designated by the National Cancer Institute. ${ }^{6}$ Before activation, the group's research protocols must pass through a rigorous peer review process and be reviewed and approved through its own committee system and the National Cancer Institute. Development of a protocol consists of six phases (box). ${ }^{6}$

Our analysis included data related to primary outcomes from all terminated phase III trials conducted by the Radiation Therapy Oncology Group since its establishment in 1968. We extracted data on methodological domains that have been acknowledged as vital 
for minimising bias in the conduct and analysis of randomised controlled trials. ${ }^{7}$ The effect of chance is usually minimised by appropriate planning of the trial's size, through a statistical power analysis using estimates for the expected differences between the interventions and prespecified type I $(\alpha)$ and type II ( $\beta)$ error levels. To investigate systematic bias we extracted data on the quality of the randomisation process (selection bias) and drop outs (attrition bias). ${ }^{8}$ Since the primary outcome was survival in most of the studies, we did not consider quality related to observer bias, such as the use of placebo or independent reading of outcomes (there were only three placebo controlled trials). We extracted data from all papers and protocols. The accuracy of this data was verified by the group's statistical centre.

\section{Results}

Overall, there were 59 terminated phase III randomised controlled trials, three of which had not been published. We found 58 published papers for the remaining 56 protocols for use in our study. The figure summarises the results according to information from the papers, protocols, and the Radiation Therapy Oncology Group's statistical office. This shows that the reporting of methods in the publications does not necessarily reflect the methodological quality of the associated protocols. For example, a priori sample size calculations were performed in $44(76 \%)$ trials, but this information was given in only nine of the 58 published papers (16\%). Although all trials had adequate allocation concealment (through central randomisation), this was reported in only $24(41 \%)$ of the papers. From our initial data extraction, we found that $40(69 \%)$ of these trials used an intention to treat analysis. This number was increased to $48(83 \%)$ after verification by the Radiation Therapy Oncology Group. End points were clearly defined, and $\alpha$ and $\beta$ errors were prespecified in $44(76 \%)$ and $43(74 \%)$ trials, respectively, but only reported in six $(10 \%)$ of the papers. Interestingly, reporting of drop outs was meticulous; we found no difference in frequency $(91 \%)$ between data presented in the papers and those in the original files.

\section{Discussion}

Poor reporting of randomised controlled trials may not indicate poor quality of the trials themselves. We are aware of two other studies that reported empirical

Phases in development of a trial protocol by Radiation Therapy Oncology Group

- Approval of concept

- Review and approval of protocol among group members

- Review by headquarters, including statistics, data management, quality assurance, protocol

administrator, and review by the institutional review board

- Review by National Cancer Institute

- Activation of protocol

- Revision of protocol

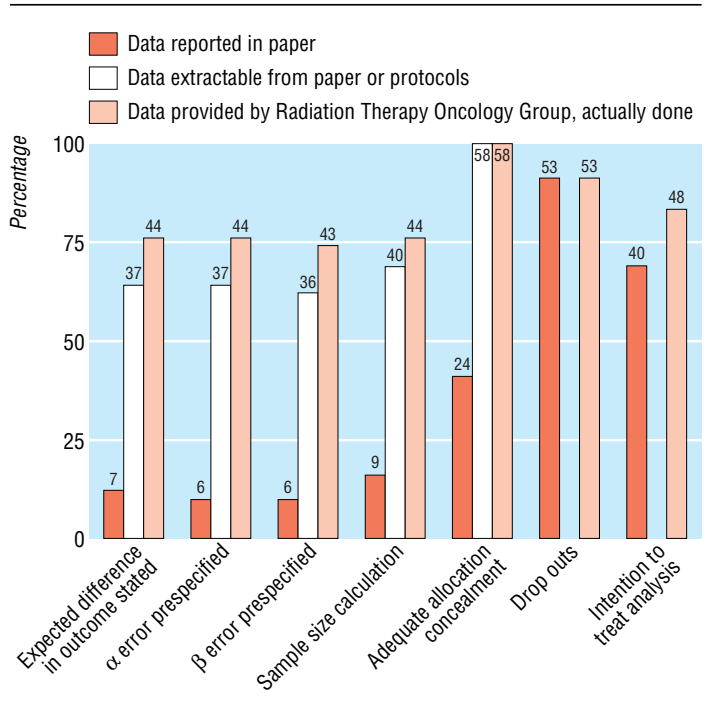

Quality of reporting compared with actual methodological quality of 56 randomised controlled trials (58 reports) conducted by the Radiation Therapy Oncology Group, based on information from published reports, protocols, and verification by the group. Absolute numbers of reports are shown

assessments of this relation. One study evaluated the quality of 63 randomised controlled trials of breast cancer treatment. Data were extracted from publications related to these trials and the results compared with the information provided by the principal investigators. The study concluded that faulty reporting reflected faulty methods. ${ }^{9}$ Another study, however, concluded that even well designed and conducted trials may be badly reported. ${ }^{2}$ This conclusion was drawn indirectly from an assessment of three key indicators of quality: adequate allocation concealment, appropriate blinding, and use of intention to treat analysis. ${ }^{2}$ Unlike our study, neither of these studies reported a comparison of the quality of reporting with the methods specified in the original research protocols.

In general, the Radiation Therapy Oncology Group and, we predict, other cooperative oncology groups sponsored by the National Cancer Institute, have conducted research of good quality. Our study is the first formal investigation of this and, we believe, the first examination of the methodological quality of randomised controlled trials performed by a cooperative oncology group.

The relation between poor reporting and poor methods was raised in 1980 in a report on patient registration, randomisation, and the importance of avoiding bias in cooperative oncology trials. ${ }^{10}$ This may have helped the cooperative oncology groups to be especially aware of methodological issues relating to trials and before the start of modern research on methodological quality. ${ }^{11}$ Consequently, for cooperative oncology groups such as the Radiation Therapy Oncology Group, even if the published description of the methods of a randomised controlled trial is poor, the quality of the trial should not be assumed to be poor. It is important to note, however, that our findings are based on a select sample of trials, which may not be representative of randomised controlled trials. Further studies to confirm the generalisability of our findings are needed and would be useful. 
Another important point relates to any assumptions that trials published before the 1996 CONSORT statement are more likely to be of poorer quality than those published after it. ${ }^{3}$ The CONSORT statement contains several methodological elements that should be followed to eliminate biased results. The intention of this statement was to improve the conduct, integrity, and reporting of randomised controlled trials. ${ }^{3}$ Our results show that studies conducted by the Radiation Therapy Oncology Group were of high quality even before publication of the CONSORT statement. It was the reports of the randomised controlled trials that showed deficiencies in their description of the methods used in the trials, not the trials themselves. Our findings indicate that although researchers in the Radiation Therapy Oncology Group were cognisant of key features in the design and conduct of good quality trials, they were less aware of the need to report these to a standard that would meet contemporary (CONSORT) requirements.

It is still appropriate to expect that the CONSORT statement will contribute to the conduct of higher quality randomised controlled trials in the future, since it incorporates and highlights many of the elements needed to perform a trial adequately. We agree with the call for all journals to adopt the policy of only publishing the report of a randomised controlled trial if it follows the CONSORT requirements. This is supported by empirical data that are now emerging about the usefulness of the CONSORT statement. For example, one study compared the quality of reports of trials before and after the CONSORT statement and found that the statement was associated with an improvement in the quality of reports. ${ }^{12}$ Further improvements in the quality of the conduct and reporting of clinical research would arise with the publication of research protocols.

Contributors: HPS and BD conceptualised the study, were involved in all aspects of the study, and wrote the first draft of the paper. SD and AK contributed to the study design, collection of data, analysis and interpretation of the data, and writing the report. MC contributed to the study design, interpretation of the data, and writing the report. CS and SS contributed to the collection of data and writing the report. BD will act as guarantor for the paper.

Funding: This research was supported by the Research Program on Research Integrity, an Office of Research Integrity/National Institute of Health collaboration, grant No 1R01NS/NR44417-01.

\section{What is already known on this topic}

Assessment of the quality of research evidence is central to informed decision making

The quality of randomised controlled trials is often based on the quality of reporting

What this study adds

Poor reporting of methods in randomised controlled trials may not reflect on poor methods themselves

Evaluation of research protocols and contacting trialists should be integral to assessing the quality of such trials

Competing interests: MC is director of the UK Cochrane Centre, which is funded by the NHS research and development programme and part of the international Cochrane Collaboration. The collaboration produces systematic reviews of health care, including randomised trials, but the views expressed here are not necessarily those of the official policy of the Cochrane Collaboration.

Ethical approval: This study was approved by the University of South Florida Institutional Review Board (No 100449).

1 Moher D, Pham B, Jones A, Cook DJ, Jadad AR, Moher M, et al. Does quality of reports of randomised trials affect estimates of intervention efficacy reported in meta-analyses? Lancet 1998;352:609-13.

2 Huwiler-Muntener K, Juni P, Junker C, Egger M. Quality of reporting of randomized trials as a measure of methodologic quality. JAMA 2002;287:2801-4

3 Moher D, Schulz KF, Altman D. The CONSORT statement: revised recommendations for improving the quality of reports of parallel-group randomized trials. JAMA 2001;285:1987-91.

4 Godlee F. Publishing study protocols: making them visible will improve registration, reporting and recruitment. BMC News Views 2001;2:4

5 Silagy CA, Middleton P. Hopewell S. Publishing protocols of systematic reviews: comparing what was done to what was planned. JAMA reviews: comparing

2002;287:2831-4. RTOG procedure manual. Philade

7 Egger M, Davey Smith G, Altman D. Systematic reviews in health care. Meta analysis in context, 2nd ed. London: BMJ Publishing, 2001.

Clarke M, Oxman A, eds. Cochrane reviewers' handbook 4.2.0 [updated March 2003]. In Cochrane Library, issue 2. Oxford: Update Software, 2003.

Liberati A, Himel HN, Chalmers TC. A quality assessment of randomized control trials of primary treatment of breast cancer. J Clin Oncol 1986; 4:942-51.

10 Herson J. Patient registration in a cooperative oncology group. Control Clin Trials 1980;1:101-10.

11 Schulz KF, Chalmers I, Hayes RJ, Altman DG. Empirical evidence of bias. Dimensions of methodological quality associated with estimates of treatment effects in controlled trials. JAMA 1995;273:408-12.

12 Moher D, Jones A, Lepage L. Use of the CONSORT statement and quality of reports of randomized trials: a comparative before-and-after evaluation. JAMA 2001;285:1992-5.

(Accepted 10 October 2003)

\section{Commentary: The quality of randomised controlled trials may be better than assumed}

Auro del Giglio, Luciano Jose Costa

ABC Foundation School of Medicine, Avenida Reboucas 3387 São Paulo, Brazil, 05410-040

Auro del Giglio chairman of haematology and oncology continued over
As readers of published articles, it is reassuring to know that the quality of published data is probably better than expected from the reporting of the methods. Soares and colleagues have addressed the discrepancies between the proposed methods in original research protocols and those reported in the final article for all 56 randomised controlled trials conducted by the Radiation Therapy Oncology Group since its creation in 1968. Good quality experimental designs were more often adhered to during the conduct of the studies than suggested by the final reports.

As readers of systematic reviews, however, we may have underestimated the quality of experimental methods, especially if reviewers had not checked the original protocols. This may have led to the exclusion of some potentially good quality papers from systematic 